\title{
STANDARDS ON AGE, HEIGHT AND WEIGHT IN OLYMPIC RUNNING EVENTS FOR MEN
}

\author{
T. KHOSLA, M.S., Ph.D. \\ Welsh National School of Medicine, Heath Park, \\ Cardiff CF 4 4XN
}

\begin{abstract}
Ideal physique in terms of height and weight is related to the type of sporting event. In an earlier communication (Khosla, 1974), it was shown that in the Olympic running events, short distance finalists are about 22 lbs $(10 \mathrm{Kg})$ heavier than long distance finalists at a standardised height of $68 \mathrm{in}$. $(173 \mathrm{~cm})$. Within a specific event there is considerable variation in the heights and weights of runners. Therefore, it is of interest to study the optimum standards on weight for height for each of the eight running events to examine if the participants and the finalists can be discriminated on the basis of criteria such as weight for height generally available for Olympic athletes.
\end{abstract}

ABSTRACT

The Olympic champions, finalists and participants are well differentiated on age, height and weight ladjusted for height) in each of the eight running events. Height for height, the champions in sprinting events are heavier than other participants but the trend is reversed in long distance events. Physiological reasons for these differences are discussed.

\section{DATA AND METHODS}

The data on the Olympic finalists from running events are derived from three volumes published by the organising committee for the Games of the XXth Olympiad, Munich 1972. The original volumes give information on date of birth, height, weight, nationality and performance of the athletes from each of the Olympic events. Age attained in September, 1972, is calculated. Height and weight are recorded in the original volumes in metric units but no indication is given on the method of measurement. International athletes know the importance of height and weight; it can reasonably be assumed that recorded heights are without shoes and weights are approximately comparable to nude weights.

For each of the events in Munich 1972, Hirate and Teipel (1973) have documented average values (without standard deviation) of age, height, weight and bulk index of participants in a paper presented at the International Meeting of Standardisation of Physical Fitness Tests. Teipel was a member of the organising committee for the Games of the XXth Olympiad, Munich 1972. The comparison of results on the participants reported by Hirate and Teipel and our results on the finalists are derived from the same source.

Key words: Olympic running events, Age, Height, Weight.
Because height and weight are correlated the comparison of body weights need adjustment for height. The body bulk index Weight/Height ${ }^{2}$ provides the most satisfactory index on the grounds that it is highly correlatied with weight and unlike other combinations of weight and height $\left(\frac{W}{H}, \frac{W}{H^{3}}\right.$ or $\left.\frac{H}{\sqrt[3]{W}}\right)$ it is uncorrelated with height; this finding has been confirmed in many international investigations (James, 1976). It should be noted that the commonly used ponderal index $\left(\frac{H}{\sqrt[3]{W}}\right)$ can lead to misleading conclusions (Khosla and Lowe, 1967). The bulk index $\frac{W}{H^{2}}$ is converted to weight at a standard height $(175 \mathrm{~cm}$; 68.9 in.) by multiplying the index by $175^{2}$ to facilitate comparisons between the various subgroups.

The bulk index $\frac{W}{H^{2}}$ does not differentiate between weight arising from muscles or from fat. Also it is an inadequate measure of somatotype in that it takes no account of body frame size. The desirable body weight standards based on the favourable mortality experience of insurance studies in the U.S.A. (Geigy, 1972) is related to height for three types of body frames:- small, medium and large. But, no indication is given on how to estimate the frame size. It is reasonable to assume that it is a man with broad chest, shoulders and pelvis, who is to be regarded as "large framed", and that "small framed" is one with a thin chest, narrow shoulders and narrow pelvis, and those intermediate are considered to be of medium frame. In an adult, changes in weight can arise from changes in controllable factors (dietary intake and exercise), whereas frame size is almost a constant feature of the individual.

\section{Definition of participants}

Participants $\left(n_{p}\right)$ referred to in the paper include the 8 finalists. If necessary mean values of "other participants" meaning non-finalists can be derived by the method of weighted averages. 


$$
\bar{x} \text { (other participants) }=\begin{gathered}
n_{p} \bar{x}_{p}-8 \bar{x}_{f} \\
n_{p}-8
\end{gathered}
$$

\section{RESULTS}

Table I compares the average values of the finalists and the participants on age, weight, height and bulk index for each of the running events. The standard deviation and ranges on each variable are also given for the finalists.

Age. In the eight running events, the finalists range in age from 20.3 years in $\mathbf{2 0 0} \mathrm{m}$ to $\mathbf{4 0 . 3}$ years in marathon (Table $I$ and Fig. 1). In the shorter distance events (100 to $800 \mathrm{~m}$ ) no finalist is observed over 30 years of age, but in the medium and long distance events (1500 or more) 10 of the 32 finalists are aged 30 years or over (Fig. 1). The figure shows that younger finalists can be found in all the running events but older finalists are found only in longer distance events. It is also observed that all the eight gold medallists in Munich were aged 26 years or below.

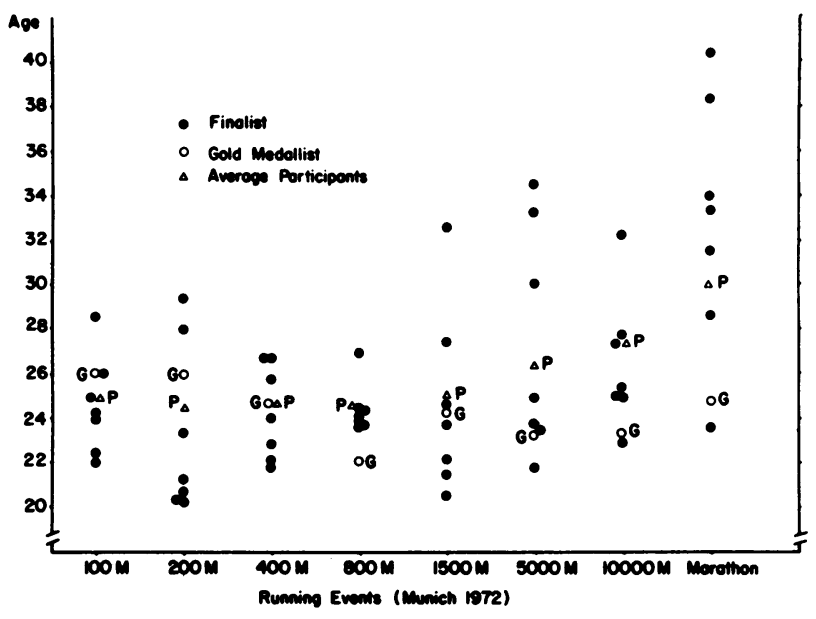

Figure 1. Age of finalists and average of participants in eight running events. The mean ages of gold medallists (Munich and Montreall are shown for comparison.

Height. Tallness confers an advantage in most of the running events. It is interesting to observe the following trends; the gold medallists laverage of Munich and Montreal) are taller than the average of the finalists, and the finalists are taller than the other participants (Fig. 2). Many other sporting events are biased in favour of the tall and interested readers are referred to earlier publications on the epidemiological aspect of this (Khosla, 1968, 1971). The average height of the U.S.A. general population (Stoudt et al, 1965), is indicated in the figure.

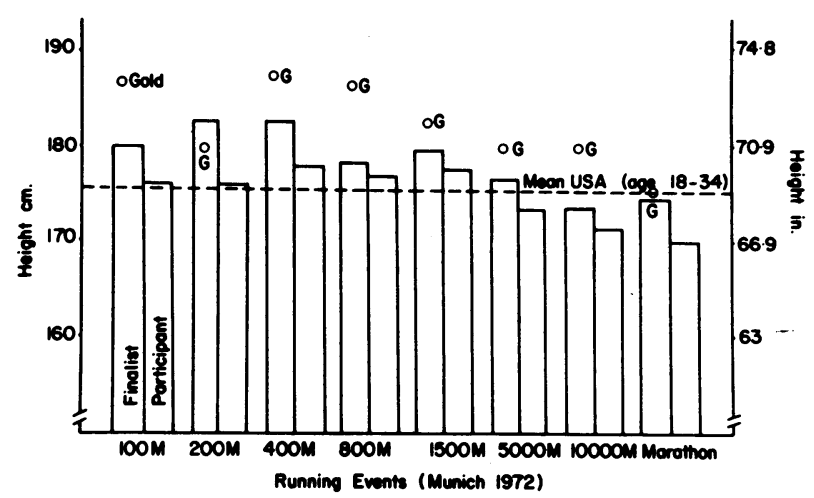

Figure 2. Average heights of gold medallists, finalists and participants in running events, and the average height of general population in the U.S.A.

Weight for Height. In the sprinting events $(100,200$, $400 \mathrm{~m}$ ) the medallists (at a standardised height) are heavier than the finalists, and the finalists are heavier than the other participants (Fig. 3). In the longer distance events, however, $(5000 \mathrm{~m}, 10000 \mathrm{~m}$ and marathon) the gold medallists (average of Munich and Montreal) tend to be lighter than the finalists, and the finalists are lighter than the other participants. In all these events, finalists and participants in general are lighter than the U.S.A. population average.

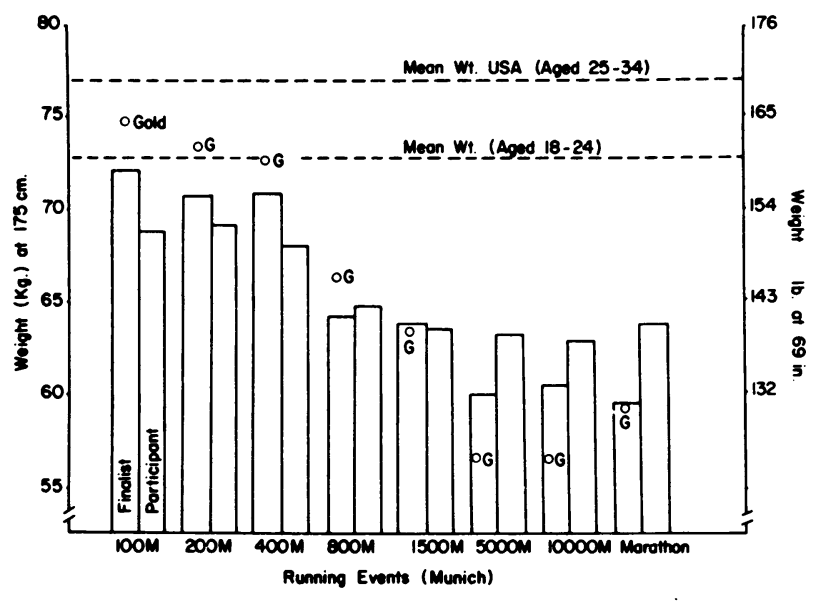

Figure 3. Average weights (adjusted to a height of $175 \mathrm{~cm}$ ) of gold medallists, finalists and participants in running events, and the average weight of general population in the U.S.A.

\section{DISCUSSION}

The highest maximal oxygen uptake is usually reached at the age of 20 (Åstrand, 1968) and yet in the long 


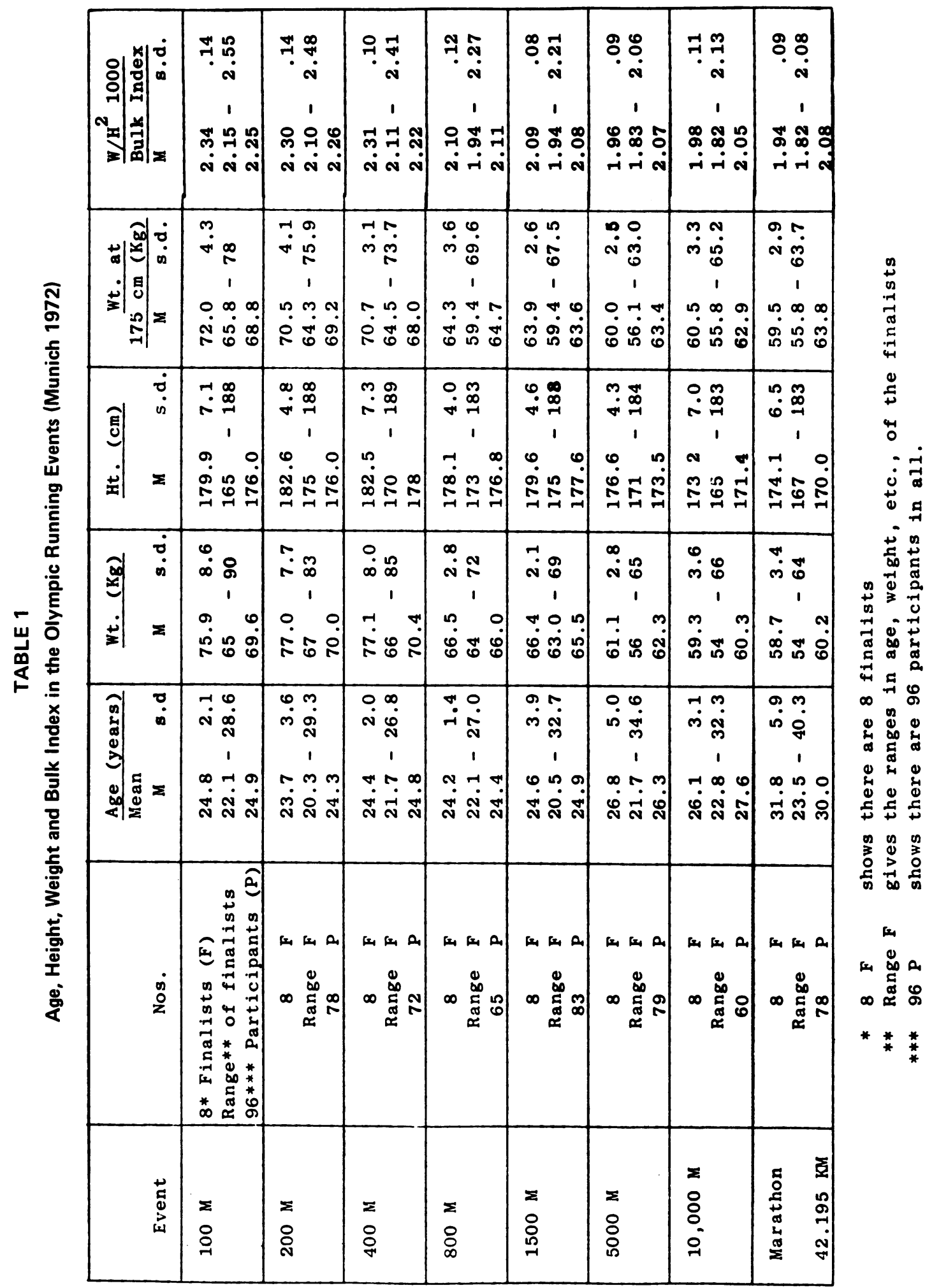


distance events some athletes are able to maintain their optimum performance over several years and indeed some champions have won gold medals in different Olympiads:-

(i) Kolehmainen, 1912, (5000 m; 10,000 m), and 1920 (Marathon).

(ii) Nurmi, $1920(10,000 \mathrm{~m})$ and $1924(1500,5000 \mathrm{~m})$.

(iii) Zatopek, $1948(10,000 \mathrm{~m}), 1952$ (5000, 10,000 and Marathon).

(iv) Abebe, 1960 and 1964 (Marathon).

(v) Viren, 1972 and $1976(5000 \mathrm{~m}, 10,000 \mathrm{~m})$.

In this connection it is of interest to quote Åstrand "... if training is continued, the maximum aerobic power can certainly be maintained and even further increased for another ten year period. Finally, the performance is also dependent on technique, tactics, motivation, and other factors, and intensive trairing and experience over the years make gradual improvement possible." In contrast, the sprinters are unable to maintain maximal performance in subsequent Olympiads.

Age is an important factor in the type of running event (Fig. 1). It appears that sprinters and medium distance runners retire at an early age around 30 years, where as the long distance runners continue running competitively well beyond their forties. The winner of a 24 hour race was aged 43, and the third position was held by a 53 year old veteran of numerous long distance races (Athletics Weekly, 1974).

Elsewhere in a different context Åstrand (1968) has demonstrated that in maximal work of short duration, a very large component of energy yield is derived from anaerobic metabolism. The relative contribution of anaerobic energy falls off as the planned period of exertion increases and conversely the relative contribution of aerobic energy rises rapidly with time (hyperbolic trend). At maximal work load of two minutes duration each contributes equally to the total energy output. In maximal work exceeding $\mathbf{3 0}$ minutes over $95 \%$ of the total energy yield is contributed by aerobic power.

In the $100 \mathrm{~m}$ race the sprinter may scarcely draw breath, but marked dyspnoea develops later. In the case of a man who ran 225 yards in 23.4 seconds, the pulmonary ventilation returned to normal in $\mathbf{2 7}$ minutes (Wright, 1953). Very severe muscular activity in the sprinting events can only be kept up for a very short time. The chemical processes going on in the body (in the absence of oxygen) yield anaerobic energy; the excess metabolites are disposed of after the exercise is over.

It is of interest to note that the champions in sprinting events derive advantages arising from excess weight compared to weights of the other participants (Fig. 3). In the medium and long distance events there is a decreasing trend towards lighter weights of the champions. Adjusting for height $(175 \mathrm{~cm})$, the average weight of the finalists in $100 \mathrm{~m}$ sprinting event $(72 \mathrm{~kg})$ exceeds the average weight of the finalists in marathon $(59.5 \mathrm{~kg})$ by $12.5 \mathrm{~kg}$; much of the excess weight in the sprinters is probably composed of muscle mass on a larger framed body. It appears that the optimum weight for height standard is related to frame size; obviously at a given height, a small framed body with a specified weight considered optimum for a large frame will become a burden to the sprinter. The choice of running event for a specified frame size is, therefore, limited.

Apart from age, height and weight very little is documented on the Olympic athlete. Many other factors of physiological and psychological nature are essential in the making of a champion. In general, possession of the right physique is not sufficient by itself, but its deviation from optimum standards in participants at the Olympic level of competitions can become a handicap to achieving excellence.

\section{REFERENCES}

Åstrand, P. 1968. Physical Performance as a function of age. JAMA 205: 11, 729.

Athletics Weekly 1974. Kent, England, November 17, 14-15.

Geigy, 1972. Scientific Tables. Ciba-Geigy Ltd., Basle, Switzerland.

Hirata, K. and Teipel, J. M. 1973. International Meeting of Standardisation of Physical Fitness Tests. Jyvaskyla, Finland, July, 22-25.

James, W. P. T. 1976. Research on Obesity. DHSS/MRC Report, HMSO, London.

Khosla, T. and Lowe, C. R. 1967. Indices of Obesity Derived from Body Weight and Height. British J.Prev.Soc.Med. 21: 122.

Khosla, T. 1968. Unfairness of Certain events in the Olympic Games. British Medical Journal 4: 111. 
Khosla, T. 1971. The Community and Sport Participation. British J.Prev.Soc.Med. 25: 114.

Khosla, T. 1974. Relationship Between Speed of Running and Distance Run. Lancet 1: 30.

Stoudt et al 1975. Weight, Height and Selected Body Dimensions of Adults . National Center for Health Statistics, Series 11, 8, U.S.A.

Wright, S., Maizels, M. and Jepson, J. B. 1953. Applied Physiology. Oxford University Press. p. 438.

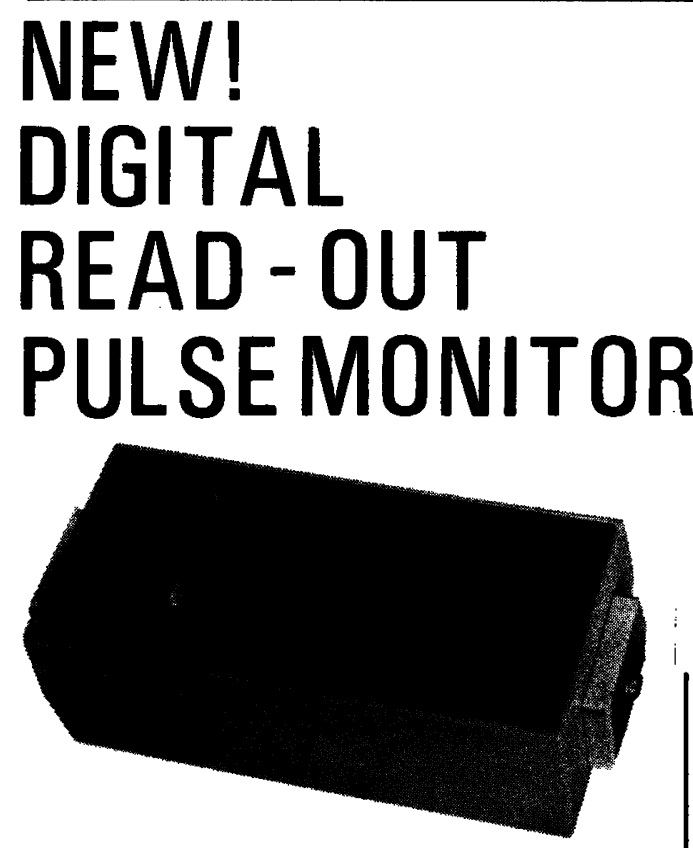

Without the use of the usual photocell finger Transducer, the instrument is simply activated by holding between both hands the integrated chrome electrodes. As long as the unit is switched ON, the digital counter calculates and displays the heartbeat per minute after 12 seconds measuring time and repeats automatically every 12 seconds showing pulse change, if any. The natural heartbeats are measured from the electrical QRS impulses between the hands, as with ECG. A set of optional chrome cylinder type hand electrodes are available and may be used in place of the integrated electrodes or, pushed onto the ends of the cycle handlebars for ergometry use. A bracket is supplied to enable the instrument to be fitted to a convenient party of the bicycle. Model LRE 012 offers many uses, from routine hospital measurements, control during fitness training; for mobile and stationary use in rehabilitation, for ergometer examinations. Power: 6v (4 x Mallory MNI400). Easy read $1 / 2$ " bright numbers. Flashing LED ORS qualitative pulse check. The instrument ensures, by the use of the most modern computer components, a real advancement in electro-medical diagnosis. Dimensions: $15 \times 7 \times 5.5 \mathrm{~cm}\left(6.5^{\prime \prime} \times 3^{\prime \prime} \times 2.2^{\prime \prime}\right)$. Weight $600 \mathrm{gm}(1.32 \mathrm{lb})$.

PRICE: $£ 125.00$ (U.K. + VAT)

\section{NEW!}
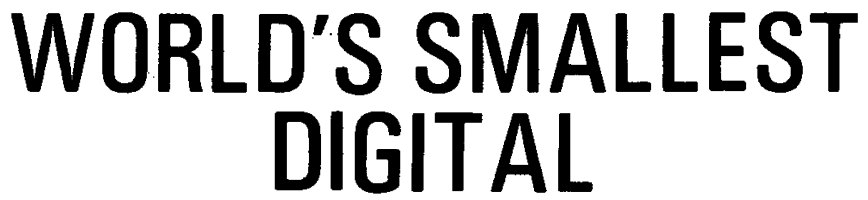

PULSE MONITOR

ALL MEDICAL USE. SPORTS MEDICINE. ALL PHYSICAL TRAINING. REHABILITATION.

LARGE LIQUID CRYSTAL DISPLAY

INEXPENSIVE DIGITAL MONITORING PULSE RATE

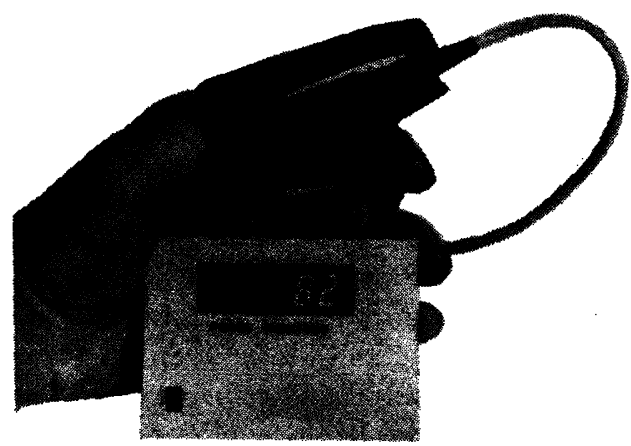

Many requests have been made over the years, for a truly miniature type Digital Heart Rate Monitor, which would be small enough to meet a multitude of applications, yet, at a keen price. We have satisfied the need for this, with the introduction of Model PM-470. Easily held in the palm of the hand or for table use, PM-470 offers fast read-out in large, bright, unmistakable LCD numbers. No fine scales to confuse the operator; no parallex to affect readings; no needle movement to falsify measurements. Running/jogging with Monitor in hand presents no problem.

Operation is simply by inserting the finger into a spring loaded Photocell Transducer and, within 20 seconds, PRB per minute is computed and displayed. Simultaneously, a flashing LCD at each pulse provides a qualitative pulse check. A special feature is AUTOMATIC REPEAT every 15 seconds, showing PRB change, if any. Controls are confined to switch ON switch OFF.

The micro Solid State electronics are contained in a unit measuring only $77 \times$ $56 \times 16 \mathrm{~mm}\left(3 \times 21 / 4 \times 5 / 8^{\prime \prime}\right)$ with a weight of $120 \mathrm{gm}$ ( $\left.4 \mathrm{oz}\right)$. The Transducer has a useful cord length of $120 \mathrm{~cm}$ (48'). Power is supplied by one 6v G413 or equivalent Silver Oxide battery, good for 15 hours continuous use, or several thousand measurements. A battery loss indicator is incorporated. The Monitor is built into a particularly tough and robust housing for hard wear and long life use. Supplied complete with Transducer, battery, carrying pouch.

PRICE $£ 99.00$ (U.K. + VAT)

STEPHENS CO., MEDICAL ELECTRONICS, 41/BASM, DICKSON ROAD, BLACKPOOL, U.K. TEL: 0253-23755 